\title{
Historia Experimental de la Química
}

\author{
Experimental history of the chemistry \\ José Antonio Chamizo
}

Facultad de Química. Universidad Nacional Autónoma de México. jchamizo@servidor.unam.mx

Recibido: 23-04-2009

\section{Resumen}

A partir de una discusión de la importancia de la experimentación en la enseñanza de las ciencias y el valor que la didáctica le da a la historia y la filosofía, se propone abordar la enseñanza experimental de la historia de la química a partir de la postura historiográfica recurrente empleando diagramas heurísticos. Lo anterior a través de diez experimentos elegidos por su valor didáctico y tecnocientífico.

\section{Palabras clave}

Historia, química, experimentación, diagramas heurísticos, modelos.

\section{Abstract}

From a discussion of the importance of experimentation in science education and the value that the didactics gives to the history and the philosophy of science, a new approach to the experimental learning of the history of the chemistry has been proposed from the recurrent historiographical position and using heuristic diagrams. The previous thing across ten experiments chosen by his didactic and technocientific value.

\section{Key words}

History, chemistry, experimentation, heuristic diagrams, models.

Un experimento más que imitar a cómo trabajan los científicos debe ser un diálogo entre el observador y el mundo natural alrededor del observador

\section{Introducción}

W. De Vos

Ante los vertiginosos cambios que enfrentan las sociedades actuales, derivados en gran medida por el impacto que las ciencias y la tecnología tienen en la vida cotidiana de sus integrantes, en los últimos años se ha presentado un intenso debate sobre otras formas de enfrentar los procesos de aprendizaje (Duschl, 1994; Matthews 1994; Just 2002). La cantidad de conocimiento científico generado es tal que no es suficiente, como se ha hecho hasta ahora, profundizar en el conocimiento espećfico de la asignatura correspondiente (por más que sin duda es fundamental). Es necesario además, incorporar la reflexión sobre la estructura de las ciencias y el papel que esta ha jugado en nuestra sociedad y, sobre todo, es necesario discutir la dinámica de cambio, puesto que lo que queremos conseguir es que los conocimientos del alumnado evolucionen hasta hacerlos rigurosos y útiles (Giere, 1988; Mc Comas, 2000). Para ello hay que escoger, de entre la enorme cantidad de información generada, aquella que permita desarrollar las competencias requeridas en un mundo cada vez más cambiante y que, por ello, prepare mejor para un futuro que no está predeterminado. Aquí hay que hacer notar que ante el enorme crecimiento de la información 
química que duplica todo lo conocido cada tres $\mathrm{o}$ cuatro años, los profesores de ciencia en general y los de química en particular (una vez que la química es la ciencia que más productiva) somos, sepámoslo o no, querámoslo o no, profesores de historia de las ciencias (Chamizo, 2007a).

En este artículo se abordará una nueva y diferente manera de aprender química, una manera en la que a partir del análisis del papel de la experimentación y de la historia de las ciencias en la didáctica, reconstruye la historia experimental de la química.

\section{La experimentación}

Este debate sobre los procesos de aprendizaje se ha extendido hacia el trabajo práctico en todas sus modalidades (Woolnough, 1985; Barbera, 1996; Izquierdo 1999, Psillos 2002, Chamizo, 2004) y que en química apela fundamentalmente a la experimentación. Dos de sus más interesantes resultados han sido, por un lado el reconocer que el propósito fundamental del mismo en la enseñanza de las ciencias es permitir que los alumnos relacionen el complejo mundo real presente con el de los conceptos construidos a lo largo de la historia, Figura 1.

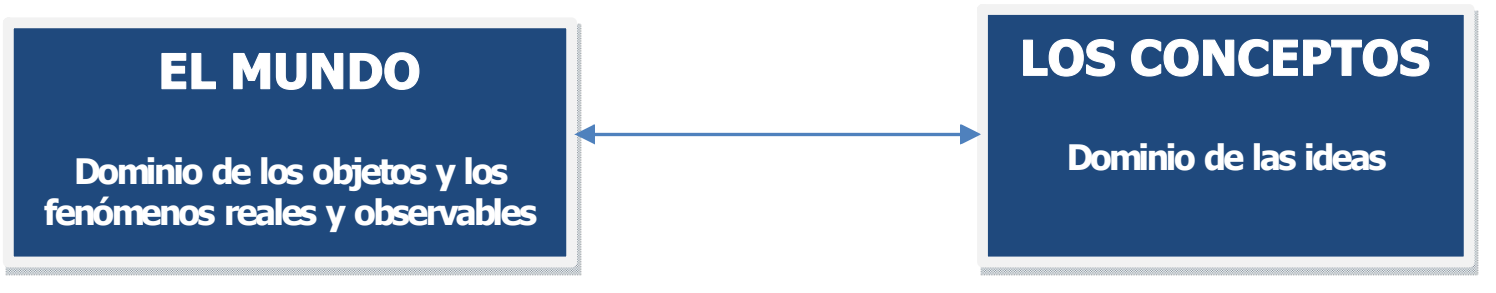

Figura 1. El propósito fundamental del trabajo práctico: permitir que los alumnos establezcan relaciones entre el mundo real y los conceptos.

Por el otro ha sido el replantearse la arbitraria distinción entre teoría, prácticas de laboratorio y problemas como algo tan diferente en la enseñanza de las ciencias que dichas actividades son impartidas a menudo por distintos profesores (Gil, 1999). Del mismo debate ha surgido un limitado acuerdo sobre los objetivos fundamentales que se persiguen al realizarlo y que aparecen como centrales en la enseñanza de las ciencias. Dichos objetivos se concretan en otras tantas actividades y son:

1 Ejercicios. Diseñados para desarrollar técnicas y destrezas espećficas.

2 Experiencias. En las que se propone que los alumnos tomen conciencia de determinados fenómenos del mundo, ya sean naturales 0 artificiales.

3 Investigaciones. En las que los estudiantes tienen que resolver un problema (que valga la redundancia para ser problema debe ser abierto).

Hay que hacer notar que un mismo fenómeno puede abordarse desde cualquiera de estas actividades y por lo tanto cumplir cualquiera de sus objetivos. Para los motivos del presente artículo me centraré exclusivamente en el trabajo práctico de investigación, aquel que más se parece a la propia investigación científica y sobre el que se han tenido intensas discusiones (Kirschner, 1988; Lewis, 2002; Caamaño, 2003; Berg, 2003; Chamizo 2007). Aquí se reconocen las ideas pioneras de Schwab (1962) sobre los 'grados de libertad' que le permitieron distinguir actividades en las que los alumnos únicamente seguían instrucciones hasta aquellas en las que ellos mismos tomaban decisiones. Herron (1971) continua en esta línea de pensamiento y es capaz de precisar los diferentes niveles que puede tener dicho trabajo (Tabla 1) en auanto a la identificación del problema a resolver, la forma en se puede solucionar y finalmente quién lo hace. 
Tecné, Episteme y Didaxis: TE $\Delta$ No. Extraordinario, 2009

$4^{\circ}$ Congreso Internacional sobre Formación de Profesores de Ciencias

Conferencias Centrales

\begin{tabular}{|c|c|c|c|}
\hline $\begin{array}{c}\text { Nivel de } \\
\text { investigación }\end{array}$ & Problema & $\begin{array}{l}\text { ¿Quién proporciona el } \\
\text { método de resolución? }\end{array}$ & Respuesta \\
\hline 0 & $\begin{array}{l}\text { Profesor / a } \\
\text { libro de texto }\end{array}$ & $\begin{array}{c}\text { Profesor / a } \\
\text { libro de texto }\end{array}$ & $\begin{array}{l}\text { Profesor / a } \\
\text { libro de texto }\end{array}$ \\
\hline 1 & $\begin{array}{l}\text { Profesor / a } \\
\text { libro de texto }\end{array}$ & $\begin{array}{l}\text { Profesor / a } \\
\text { libro de texto }\end{array}$ & Alumna/ o \\
\hline 2 & $\begin{array}{l}\text { Profesor / a } \\
\text { libro de texto }\end{array}$ & Alumno/ a & Alumna/ o \\
\hline 3 & Alumno/ a & Alumno/ a & Alumna/ o \\
\hline
\end{tabular}

Tabla 1.Marco de análisis para determinar el nivel de investigación de un trabajo práctico (Herron, 1971; Tamir, 1989).

Como se puede observar en dicha tabla la inmensa mayoría del trabajo práctico que se realiza en las escuelas corresponde, cuando se lleva a cabo una investigación, a los niveles 0 y 1 . Los problemas y las formas de resolverlos son ajenas a los estudiantes que se convierten así en meros espectadores de su potencial propio aprendizaje. Lo anterior contrasta con lo expuesto por Hodson (1994) en el sentido de que los estudiantes aprenden mejor si son activos, en lugar de pasivos.

\section{La historia}

La historia en general y la de las ciencias en particular nos ha enseñando que las que fueron respuestas correctas para preguntas de su tiempo, años después fueron consideradas erróneas. Los héroes se convirtieron en villanos y viceversa. No hay verdades absolutas: «las ciencias son más un viaje que un destino».

El historiador H. Kragh (1987) reconoce al menos tres posturas en el quehacer histórico, tres diferentes estrategias historiográficas: anacrónica, diacrónica y recurrente.

En la estrategia anacrónica el pasado se estudia y se valida de manera "absoluta" a la luz del presente. Esta postura etiquetada con el nombre de interpretación "whig" ha sido ampliamente utilizada y también muy cuestionada. Por otro lado la estrategia diacrónica consiste en estudiar la ciencia del pasado de acuerdo a las condiciones que existían realmente en ese pasado. Sin embargo el mismo Kragh indica: La historiografía diacrónica no puede ser más que un ideal. El historiador no puede liberarse de su tiempo ni evitar completamente el empleo de patrones contemporáneos.

Más recientemente Tosh (2003) argumenta que la historia de la ciencia es inherentemente "centrada en el presente" asunto que de otra manera propuso hace años el filosofo francés G. Bachelard (1972) cuando introdujo el término "historia recurrente de la ciencia" como aquella que es continuamente contada a la luz del presente. El objetivo de la historia recurrente no consiste en encontrar los conceptos que actualmente usamos en algún punto del pasado, sino el revelar el camino por el aul esos conceptos emergieron a partir de otros conceptos en una secuencia de correcciones y rectificaciones. Cuando un nuevo concepto "aparece" introduce una reorganización de la disciplina en la cual se incorpora y una evaluación del conocimiento previo con que esta contaba. Desde este punto de vista la ciencia se compromete periódicamente a evaluarse a si misma, a reconocerse en su pasado. Esta historia recurrente es deliberadamente anacrónica, pues decide si la ciencia anterior es válida, o no, a la luz de los conocimientos actuales.

La reconstrucción racional a la que apela la historia recurrente es diferente de la propuesta por Lakatos (1978) en la cual se tiene como referencia absoluta un standard extrahistórico de racionalidad. En este sentido es más cercana a la propuesta de Toulmin (1972) y su propuesta de racionalidad moderada: 
Las cuestiones de racionalidad conciemen precisamente no a las doctrinas intelectuales particulares que un hombre -o un grupo profesional- adopta en cualquier momento dado, sino a las condiciones y la manera en que está dispuesto a criticar $y$ modificar esas doctrinas a medida que pasa el tiempo. La racionalidad de una ciencia no está encamada en los sistemas teóricos conientes en ella en momentos determinados, sino en sus procedimientos para llevar a cabo descubrimientos y cambios intelectuales a través del tiempo.

Así una herética revisión sobre la historia de las ciencias y la tecnología indica que ésta no se refiere únicamente a una serie de sucesiones, o de reemplazos de una clase de conocimiento por otra; es más una cuestión de acumulación compleja y de variedad simultánea, disputada en un cierto plazo. Compartiendo la postura del historiador de la ciencia inglés J. Pikestone (Pickstone, 2000) aquí se reconocen tres grandes tipos de formas de conocer:

> La historia natural que se refiere a una primera dasificación de los componentes del mundo. Comprende la variedad de objetos naturales o artificiales, normales o patológicos. Es el espacio de las taxonomías: celestes, geológicas o biológicas y del lugar donde se vuelven públicas, los jardines botánicos, los zoológicos y los grandes museos de ciencias.

> El análisis. Si la variedad y el cambio son identificados por la historia natural, el análisis busca el orden por disección. El análisis aparece cuando los objetos se pueden ver como compuestos de 'elementos', o auando los procesos se pueden ver como el 'flujo' de un 'elemento ' a través de un sistema. Es el espacio de los laboratorios de anatomía, química, física e ingeniería y del lugar donde se vuelven públicos, las escuelas, institutos, politécnicos, hospitales y universidades.

> La síntesis. Si el análisis considera el separar cosas, la síntesis es sobre cómo ponerlas juntas. El análisis especifica la composición de lo 'conocido' para posteriormente, poniendo juntos los 'elementos', crea nuevos objetos o fenómenos. La experimentación se basa en la síntesis, en la producción sistemática de la novedad. Es el espacio 'privado' del control, ya sea por motivos militares o económicos, de los laboratorios de biomedicina y farmacia, de diseño de nuevos materiales o de física nudear, así como del lugar donde se vuelven públicos, el complejo tecnocientúfico industrial.

Estas tres grandes formas de conocer, que no necesariamente son consecutivas, sirven de marco de referencia en la presentación histónica experimental de la química faltando por indicar como se desarrolla una determinada disciplina. La postura aquí adoptada, de entre varias posibles, es a través de la resolución de problemas.

Aceptando el papel central de los problemas en el avance de la ciencia, el filósofo inglés $\mathbf{S}$. Toulmin (Toulmin, 1972, Chamizo, 2007b) avanza en la caracterización de los problemas a través de la expresión:

\section{Problemas = ideales explicativos - capacidades corrientes}

Así, los problemas se presentan en la "distancia" o "diferencia" que hay entre aquello a lo que una comunidad o un individuo aspira a comprender (lo que se denomina ideales explicativos) y la capacidad que tiene esa comunidad o individuo para alcanzarlo; esta distancia se acorta o desaparece auando emergen nuevos conocimientos. Ahora bien, esa aspiración se concreta generalmente a través de preguntas y las preguntas que concretan los problemas son preguntas de su tiempo (Tabla 2). Sobre la combustión, por ejemplo, no se preguntó lo mismo el francés A. Lavoisier hace más de doscientos años (sus preguntas estaban centradas en la existencia e importancia del oxígeno en dicho fenómeno) que el premio Nobel de Química en 1986, de origen 
chino, Yuan T. Lee (aryas preguntas se centraban en las más de cien sustancias que se encuentran en una llama). Las preguntas y sus respuestas, los modelos (siempre tentativos) y el lenguaje que se emplean para explicarlas generan conocimiento que se aplica y modifica el mundo.

> La necesidad de un contexto. Toda pregunta debe estar contextualizada, es decir debe contener indicadores explícitos, tanto históricos como geográficos, derivados de los hechos.

> La necesidad de dar indicios sobre los conceptos implicados. Es dear dar indicios sobre los modelos, el lenguaje y/o las aplicaciones relacionadas en ese lugar y en esa época.

> La necesidad de dar indicios sobre la metodología. No necesariamente experimentales y actuales. Las respuestas a muchas preguntas pueden obtenerse a través de otro tipo de metodologías.

Tabla 2 Recomendaciones para formular buenas preguntas

Así, los conceptos que ahora se aprenden en las dases de ciencias son el resultado de muchas preguntas, de problemas resueltos y de aplicaciones más o menos afortunadas que fueron enigmas en su momento. En pocas palabras los conceptos son construcciones históricas.

Los conceptos científicos, cambiantes a lo largo de la historia, integran una complejidad tal, que es necesario distinguir en ellos tres características o dimensiones que, así como permitieron construirlos, van a permitir utilizarlos: el lenguaje, las técnicas de representación y los procedimientos de aplicación de la ciencia. Los dos primeros se refieren a aquellos aspectos simbólicos de la explicación cientúfica -esto es, la actividad científica que llamamos explicar-, una de las formas en las que hacemos públicos nuestros pensamientos, una de las formas en las que una generación le transmite a otra el contenido de una ciencia, una «enculturación». Estos solo tienen un uso genuinamente explicativo cuando se aplican en el mundo. Así el tercer aspecto comprende el reconocimiento de situaciones a las que son apropiadas estas actividades simbólicas, el entomo.

\section{Historia experimental de la química}

La postura historiográfica recurrente y estas tres grandes formas de conocer, que no necesariamente son consecutivas, sirven de marco de referencia en la presentación histórica experimental de la química.

Antes de abordar ese asunto es necesario hacer notar el reciente debate sobre los experimentos más bellos en la historia de la química.

La American Chemical Society (ACS) a través de su revista Chemical and Engeeniering Neus convocó a sus lectores a escoger los experimentos más bellos en la historia de la química (Freemantle, 2003). De las 70 respuestas se identificaron 55 experimentos que recibieron al menos dos votos y solamente cinco experimentos recibieron más de tres votos. Los editores de la revista eligieron entonces a los 25 experimentos más nominados y los enviaron a un conjunto previamente seleccionado de historiadores y químicos, con la tarea de que identificaran los 10 mejores. Dicha lista en orden decreciente se presenta en la Tabla 3.

Por otro lado dos años después, la británica Royal Society publicó el texto de P.Ball, Elegant Solutions. Ten beautiful experiment in chemistry. Aquí el autor, conocedor de la lista publicado por la ACS, se concentra más en el sentido del experimento y de la belleza privilegiando atributos como la invención, la elegancia, la perseverancia o la imaginación y cuestiona el que Lavoisier haya realizado un experimento o que Perkin se induya en dicha lista ya que su trabajo fue fruto del azar.

Mi aproximación es otra. Privilegio la historia en función de la didáctica (Gallego, 2007). Así 
construí una lista, también de diez experimentos, a través de los cuales se pueda contar la historia de la química de manera cronológica y que puedan ser reconstruidos con aparatos modemos en un laboratorio escolar de seaundaria. bachillerato o primer año de universidad. Por eso mismo algunas de las metodologías para resolver las preguntas que dichos experimentos pueden responder no requieren mas de dos horas para llevarse a cabo. Adicionalmente los experimentos, enfrentados como investigaciones, permiten considerar la dasificación de Pickstone, es decir pueden discutirse como historia natural, análisis o síntesis.

\begin{tabular}{|c|c|c|}
\hline Freemantle (2003) & Ball (2005) & Chamizo (2009) \\
\hline $\begin{array}{l}\text { Pasteur: } \quad \text { separación } \\
\text { enantiómeros (1848) }\end{array}$ & $\begin{array}{l}\text { Van Helmont: el sauce y la } \\
\text { belleza de la cuantificación }\end{array}$ & $\begin{array}{l}\text { Los metales de la antigüedad. EI } \\
\text { cobre }\end{array}$ \\
\hline $\begin{array}{l}\text { Oxidación: de los metales por } \\
\text { Lavoisier (1775) }\end{array}$ & $\begin{array}{l}\text { Cavendish: el agua y la belleza } \\
\text { del detalle }\end{array}$ & $\begin{array}{l}\text { La destilación, técnica } \\
\text { desarrollada por los alquimistas } \\
\text { árabes }\end{array}$ \\
\hline $\begin{array}{l}\text { Fisher: determinación de la } \\
\text { configuración de la glucosa (1890) }\end{array}$ & $\begin{array}{l}\text { Los esposos Curie: el radio y la } \\
\text { belleza de la paciencia }\end{array}$ & $\begin{array}{l}\text { Los metales se pueden quemar. P. } \\
\text { Brun y J. Rey }\end{array}$ \\
\hline $\begin{array}{l}\text { Dayy: aislamiento de metales } \\
\text { alcalinos y alcalinoterreos usando } \\
\text { electrolisis (1808) }\end{array}$ & $\begin{array}{l}\text { Rutherford: las partículas alfa y } \\
\text { la belleza de la elegancia }\end{array}$ & $\begin{array}{l}\text { La combustión para demostrar } \\
\text { que el aire es una mezda. J. } \\
\text { Mayow }\end{array}$ \\
\hline Perkin: colorantes de anilina (1856) & $\begin{array}{l}\text { Seaborg: el seaborgio y la } \\
\text { belleza de lo pequeño }\end{array}$ & $\begin{array}{l}\text { Del aire desflogisticado de J. } \\
\text { Priestley al oxígeno de A. Lavoisier }\end{array}$ \\
\hline $\begin{array}{l}\text { Kirchoff y Bunsen: lineas } \\
\text { espectrales (1859) }\end{array}$ & $\begin{array}{l}\text { Pasteur: los cristales y la belleza } \\
\text { de la simplicidad }\end{array}$ & $\begin{array}{l}\text { La descomposición del agua por } \\
\text { electrólisis. W. Nicholson }\end{array}$ \\
\hline $\begin{array}{l}\text { Prietley: descubrimiento del } \\
\text { oxígeno (1774) }\end{array}$ & $\begin{array}{l}\text { Urey y Miller: la química } \\
\text { prebiótica y la belleza de la } \\
\text { imaginación }\end{array}$ & $\begin{array}{l}\text { La industria de los colorantes. W. } \\
\text { Perkin }\end{array}$ \\
\hline $\begin{array}{l}\text { Bartlett: Preparación de } \\
\text { compuestos de gases nobles (1962) }\end{array}$ & $\begin{array}{l}\text { Bartlett: la química del xenón y } \\
\text { la belleza de lo sencillo }\end{array}$ & 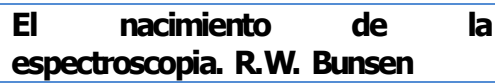 \\
\hline 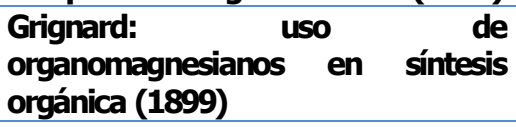 & $\begin{array}{l}\text { Woduard: la vitamina } B_{12} \text { y la } \\
\text { belleza de la economía }\end{array}$ & $\begin{array}{l}\text { La síntesis de la Aspirina. F. } \\
\text { Hoffman }\end{array}$ \\
\hline $\begin{array}{l}\text { Los esposos Curie: Descubrimiento } \\
\text { de elementos radiactivos (1898) }\end{array}$ & $\begin{array}{l}\text { Paquette: el dodecaedrano y la } \\
\text { belleza del diseño }\end{array}$ & $\begin{array}{l}\text { El nylon y las macromoléculas. } \\
\text { W.H. Carothers }\end{array}$ \\
\hline
\end{tabular}

Tabla 3. Diez experimentos en la historia de la química.

Como se observa en la Tabla 3, al menos tres de estos experimentos didácticos coinciden con las listas previamente publicadas. Es complejo validar los otros siete además de lo dicho anteriormente pero daré algunos indicios. El aislamiento de los metales fue fundamental en la historia de las sociedades humanas como también de primordial importancia lo fue el desarrollo de la destilación por los alquimistas del Islam. No nos reconoceríamos sin artículos metálicos, bebidas alcohólicas, ni perfumes. Pero tampoco sin saber que los metales se pueden quemar...y se queman. Y cuando esto sucede sus propiedades cambian preparando el entendimiento de la reacción química. La lenta pero inexorable ruptura del aristotélico elemento "aire" en una confusa mezcla de gases permitió primero aislar y luego caracterizar nuevos elementos. Con el advenimiento de las pilas eléctricas, la historia natural de la química se pobló, ahora si, de nuevos elementos producidos por la ruptura de diversos materiales (entre ellos el antiguo elemento agua). El azar llevó a Perkin a descubrir los colorantes artificiales y con ello se consolidó la industria química moderna a lo que contribuyó la compleja pelea por la patente de la aspirina, la droga universal. Finalmente resulta imposible pensar la mecánica cuántica sin la espectroscopía, ni la modemidad material que se esbozaba en la primera mitad del siglo XX sin los polímeros.

De lo anterior queda daro que, en lugar de los comunes reportes de práctica de laboratorio es posible utilizar diagramas heurísticos, que no son otra cosa que Ves de Gowin modificadas (Chamizo, 2007) para los trabajos prácticos de investigación, Figura 2 Es decir se asume que los 
diez experimentos que se presentan pueden abordarse como investigaciones cuya profundidad y complejidad dependerá de cada estudiante o grupo de estudiantes. Así, en una sola página, hay que indicar los hechos históricos que se conocáan en ese momento (documentándolos) y sobre los arales se construye una pregunta (Tabla 2). La respuesta a la misma requiere de dos acciones. Por un lado una metodología (sección derecha del diagrama) que inicia por el establecimiento de que hacer para responder a la pregunta, hacerlo y analizar los resultados obtenidos. Por el otro (sección izquierda del diagrama) utilizar los conceptos que se conocían en la época, es decir las aplicaciones, el lenguaje y los modelos de explicación y los que se utilizan en la actualidad (historiografía recurrente). La complejidad requerida sugiere que sólo se enuncien los asuntos más significativos siendo necesario, desde luego, comprenderlos. Utilizando tanto la metodología como los conceptos es posible llegar a una respuesta.

\section{Diagrama Heurístico (ejemplo)}

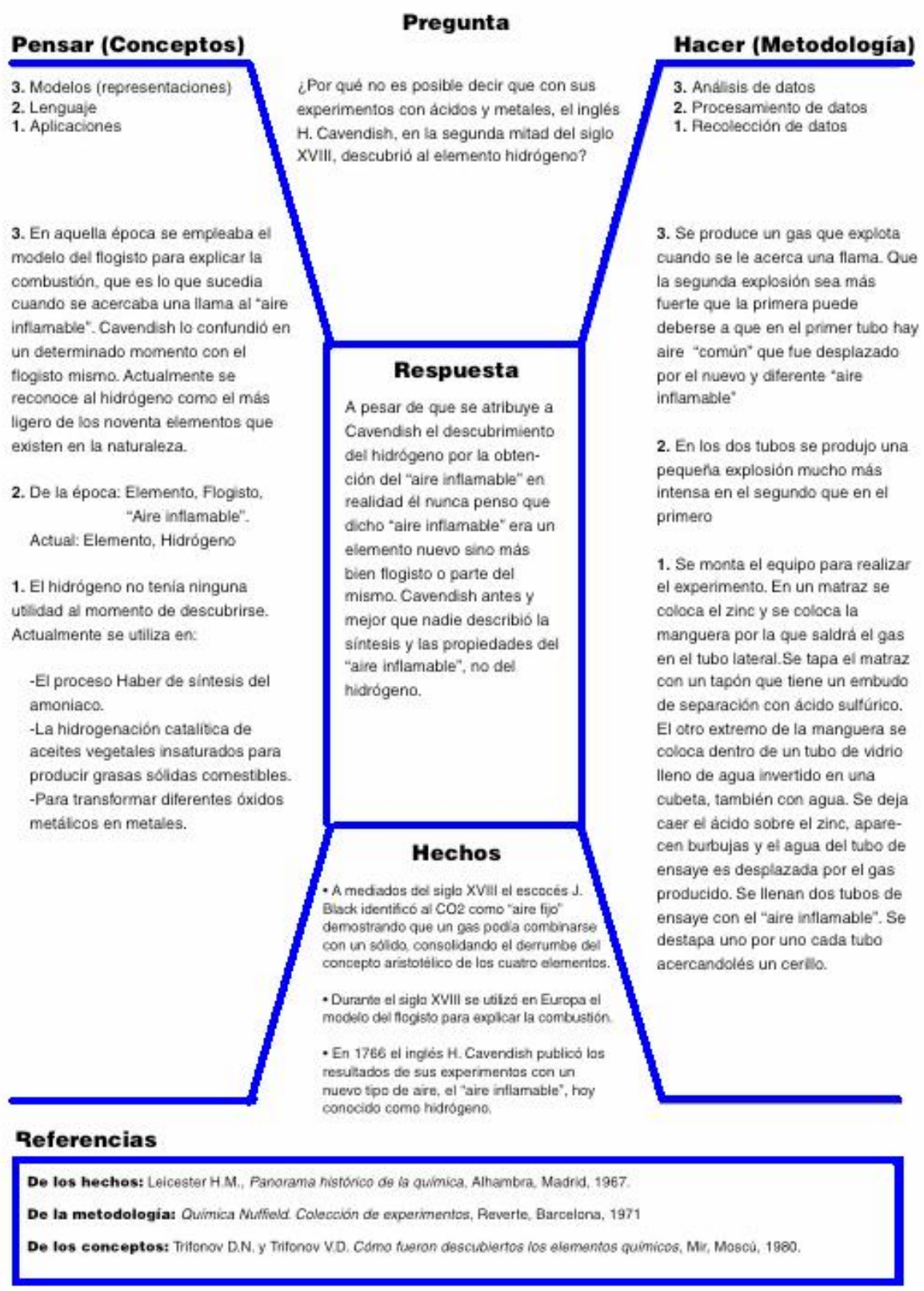

Figura 2. Diagrama heurístico sobre el descubrimiento del hidrógeno. 


\section{Conclusiones}

Una primera experiencia de esta aproximación didáctica se ha llevado a cabo en la Facultad de Química de la UNAM y de la misma queda claro, de manera preliminar, que es posible enseñar química de otra manera.

Hace casi una década Justi (2000) indicó la importancia de utilizar modelos históricos en la enseñanza de la ciencia. La presente propuesta acepta su sugerencia pero difiere de la de ella en que se soporta, para su empleo en el salón de dases, en las ideas de recurrencia de Bachelard y de desarrollo histórico de Toulmin, incorporando además como henramienta de trabajo los diagramas heurísticos.

Así, de lo aquí presentado y como lo indica el epígrafe, es posible enseñar historia, e historia de la química, propiciando el diálogo entre los alumnos y el mundo que les rodea, reflexionando y realizando algunos de sus experimentos más significativos.

\section{Bibliografía}

Bachelard, G., (1972). El compromiso racionalista, Buenos Aires, Siglo XXI.

Ball P. (2005). Elegant Solutions. Ten beautiful experiments in chemistry, London, RSC.

Barbera O, y Valdés P., (1996). El trabajo práctico en la enseñanza de las ciencias: una revisión, Enseñanza de las ciencias, 14, 365-379.

Berg C.A R, Bergendahl C. B., Lundberg B.K.S. (2003). Benefiting from an open-ended experiment? A comparision of attitudes to, and outcomes of, an expository versus an open-inquiry version of the same experiment, International Joumal of Science Education, 25, 351-372

Caamaño A. (2003). Los trabajos prácticos en ciencias, en Jiménez Aleixandre (coord.) Enseñar ciencias, Barcelona, Grao.

Chamizo J.A. (compilador) (2004). Antología de la Enseñanza Experimental, UNAM, México.

Chamizo J.A. e Izquierdo M, (2007). Evaluación de las competencias de pensamiento científico, Alambique, 51, 9-19.

Chamizo J.A. (2007a). Teaching modem chemistry through 'recurrent historical teaching models, Science\&Education, 16, 197-216.

Chamizo J.A (2007b). Las aportaciones de Toulmin a la enseñanza de las ciencias. Enseñanza de las ciencias, 25, 133-146.

Chamizo J.A. (2009). Introducción a la historia experimental de la química, México, FQ-UNAM.

Duschl, R.A. (1994). Research in the History and Philosophy of Science, in D.L Gabel (ed.), Handbook of Research on Science Teaching and Learning, New York, Macmillan.

Freemantle M. (2003). Chemistry at its most beautiful, C\&ENeus, 25 august. 81, 34, 27-30.

Gallego A. (2007). Ciencia, historia, epistemología y didáctica de las ciencias: las comunidades de especialistas, Tecné, Episteme y Didaxis, 22, 113-125. 
Giere R. (1988) Explaining Saience. A cognitive approach, Chicago, University of Chicago Press.

Gil D., Furio C., Valdés P., Salinas J., Martínez-Torregrosa J., Guisáosla J., González E., DumasCarre A., Goffard M. y Pessoa A, (1999). ¿Tiene sentido seguir distinguiendo entre aprendizaje de conceptos, resolución de problemas de lápiz y papel y realización de prácticas de laboratorio? Enseñanza de las ciencias, 17, 311-319.

Herron D., (1971). The nature of scientific enquiry', school Science Review, 79, 171-172

Hodson D. (1994). Hacia un enfoque más crítico del trabajo experimental, Enseñanza de las ciencias, 12, 299-313.

Izquierdo M, Sanmartí N. y Espinet M., (1999). Fundamentación y diseño de las prácticas escolares de ciencias experimentales, Enseñanza de las ciencias, 17, 45-59.

Justi R, (2000), Teaching with Historical Models in Gilbert J.K. and Boutler C.J. eveloping Models in Science Education, Dordrecht, Kluwer.

Justi, R. and Gilbert, J. (2002). Models and Modeling in Chemical Education in Chemical Education:Towards Research-based Practice, Dordrecht Kluwer.

Kragh H. (1987). An Introduction to the Historiography of Science, Cambridge, Cambridge University Press.

Kirshner P. and Meester M, (1988). Laboratory approaches', Higher Education, 17, 81-98.

Lakatos I. (1978). Mathematics, Science and Epistemology: Philosophical papers 2 Worral J. and Currie G (eds), Cambridge, Cambridge University Press.

Lewis J. (2002). The Effectiveness of Mini-Projects as a Preparation for Open-ended Investigations, in Psillos D. and Niedderer (eds), Teaching and learning in the science laboratory, Dordrecht, Kluwer Academic Publishers.

McComas W.F., (2000), The Nature of Science in Science Education. Rationales and Strategies, Dordrecht, Kluwer Academic Publishers.

Matthews, M.R. (1994). Science Teaching: The Role of History and Philosophy of Saience, London, Routledge.

Millar R., Tiberghein A. and Le Maréchal J.F., (2002). Varieties of Labwork: a Way of Profiling Labwork Tasks in Psillos D. and Niedderer (eds), Teaching and learning in the science laboratory, Dordrecht, Kluwer Academic Publishers, Dordrecht

Pickstone J.V. (2000). Ways of knowing, Manchester, Manchester University Press.

Psillos D. and Niedderer (eds), (2002). Teaching and leaming in the science laboratory, Dordrecht, Kluwer Academic Publishers.

Tamir P. (1989). Training Teachers to Teach Effectively in the Laboratory. Science Education 73, 59-69.

Tosh N., (2003). Anachronism and retrospective explanation: in defense of a present-centered history of science, Stud.Hist Phil.Sai, 34, 647-659 
Tecné, Episteme y Didaxis: TE $\Delta$ No. Extraordinario, 2009

$4^{\circ}$ Congreso Intemacional sobre Formación de Profesores de Ciencias

Conferencias Centrales

Toulmin S. (1972) Human Understanding, Princeton, Princeton University Press.

Woolnough, B.E., and Allsop T., (1985). Practical work in science, Cambridge Cambridge University Press. 